\title{
ARTIGO 15
}

\section{IDENTIFICAÇÃO DOS CONHECIMENTOS DE MÃES NA PREVENÇÃO DE ACIDENTES DOMÉSTICOS COM CRIANÇAS DA PRIMEIRA INFÂNCIA}

Essyo Pedro Moreira de Lima ${ }^{1}$, Adrielle Oliveira Azevedo de Almeida², Eveline Pinheiro Beserra ${ }^{2}$, Elioneide Paulo Carneiro ${ }^{2}$, Francisco Mairton Rodrigues de Andrade², Fabiane do Amaral Gubert ${ }^{2}$

Objetivo: identificar os conhecimentos das mães sobre a prevenção de acidentes domésticos infantis com crianças da primeira infância. Metodologia: trata-se de um estudo descritivo-exploratório, com abordagem quantitativa. Foi realizado em um ambulatório de puericultura localizado no Campus do Pici da Universidade Federal do Ceará, na cidade de Fortaleza. A amostra foi constituída por 32 mães. Resultados: o estudo demonstrou que há predominância de acidentes domésticos infantis em populações de baixa renda, escolaridade média, idade jovem e com várias pessoas morando na mesma residência. Percebeuse a necessidade de serem fortalecidos conhecimentos junto as mães sobre prevenção de acidentes domésticos infantis, objetivando assim prevenir a ocorrência de injúrias, podendo ser criados para este fim programas e ações de prevenção. Conclusão:percebeu-se ainda que as abordagens prevencionistas não devem ser pautadas apenas nos agentes lesivos, visto que as variáveis levantadas influenciam umas às outras, direta ou indiretamente, na ocorrência de acidentes domésticos.

Descritores: Prevenção de Acidentes; Enfermagem Pediátrica; Cuidados de Enfermagem.

\section{IDENTIFICATION OF KNOWLEDGE OF MOTHERS IN THE PREVENTION OF DOMESTIC ACCIDENTS WITH CHILDREN OF THE FIRST CHILDHOOD}

Objective: to identify mothers' knowledge about the prevention of childhood domestic accidents with infants. Methodology: this is a descriptive-exploratory study, with a quantitative approach. It was carried out in a pediatric outpatient clinic located at the Pici Campus of the Federal University of Ceara, in the city of Fortaleza. The sample consisted of 32 mothers. Results: the study showed that there is a predominance of childhood domestic accidents in low income, middle schooling, young age and several people living in the same household. It was noticed the need to strengthen the knowledge of mothers on the prevention of domestic accidents for children, in order to prevent the occurrence of injuries, and prevention programs and actions could be created for this purpose. Conclusion: it was also noticed that preventive approaches should not be based only on the harmful agents, since the variables raised influence directly or indirectly on the occurrence of domestic accidents.

Descriptors: Accident Prevention; Pediatric Nursing; Nursing Care.

\section{IDENTIFICACIÓN DE LOS CONOCIMIENTOS DE MADRE EN LA PREVENCIÓN DE ACCIDENTES DOMÉSTICOS CON NIÑOS DE LA PRIMERA INFANCIA}

Objetivo: identificar los conocimientos de las madres sobre la prevención de accidentes domésticos infantiles con niños de la primera infancia. Metodología: se trata de un estudio descriptivo-exploratorio, con abordaje cuantitativo. Se realizó en un ambulatorio de puericultura ubicado en el Campus del Pici de la Universidad Federal de Ceará, en la ciudad de Fortaleza. La muestra fue constituida por 32 madres. Resultados: el estudio demostró que hay predominio de accidentes domésticos infantiles en poblaciones de bajos ingresos, escolaridad media, edad joven y con varias personas que viven en la misma residencia. Se percibió la necesidad de ser fortalecidos conocimientos junto a las madres sobre prevención de accidentes domésticos infantiles, con el objetivo de prevenir la ocurrencia de injurias, pudiendo ser creados para este fin programas y acciones de prevención.Conclusion: Se percibió también que los enfoques prevencionistas no deben ser pautados sólo en los agentes lesivos, ya que las variables levantadas influencian unas a otras, directa o indirectamente, en la ocurrencia de accidentes domésticos.

Descriptores: Prevención de Accidentes; Enfermería Pediátrica; Cuidados de Enfermería 


\section{INTRODUÇÃO}

A primeira infância, etapa reconhecida como os primeiros anos de vida de um ser humano, em particular os três primeiros, é marcada por uma fase determinante para a capacidade cognitiva, emocional e social do indivíduo, pois o cérebro absorve todas as informações, e as respostas são rápidas e duradouras. Destaca-se que é nessa fase em que as crianças são mais vulneráveis às situações de acidentes, mais do que em outros períodos do desenvolvimento(1).

Nesse período, quaisquer acontecimentos externos, como, por exemplo, acidentes, podem afetar diretamente o crescimento e o desenvolvimento humanos. Quanto menor a idade da criança, menor sua percepção de risco e maior sua vulnerabilidade e dependência de terceiros em termos de segurança contra acidentes e desastres ${ }^{(2)}$.

Os acidentes domésticos são situações complexas, não intencionais e evitáveis em sua maioria. Além de provocarem custos sociais, econômicos e emocionais, são também responsáveis por sequelas e eventos fatais que, a longo prazo, repercutem na família e na sociedade, penalizando crianças e adolescentes $^{(3)}$.

Os acidentes - dentre eles queda, queimadura. sufocamento, afogamento, intoxicação, envenenamento, agressão física, asfixia, cortes, eletrocussão, escoriações, esmagamentos, mordeduras e perfurações - ocorrem em muitos lares, independentemente do nível social ou intelectual dos individuos, nem sempre significando negligência por parte dos protetores, podendo ocasionar hospitalização acompanhada ou não de acometimento e até da morte da criança ${ }^{(4.5)}$.

Segundo uma pesquisa realizada no Brasil com 7.224 crianças de 0 a 9 anos, a partir de um levantamento dos atendimentos em unidades de urgência e emergência em 24 capitais brasileiras e no Distrito Federal, foram evidenciadas 181 internações por violência e 7.043 por acidentes, dos quais $67,9 \%$ ocorreram no ambiente domiciliar, sendo $52,4 \%$ por meio de queda, 31,7\% resultando em contusão/ entorse/ luxação e $42,6 \%$ atingindo cabeça/ pescoço(6).

Em uma pesquisa feita em Salvador-BA, com 609 individuos de 196 famílias de baixa renda objetivando medir a ocorrência de acidentes nos últimos 15 dias, mostrou que 58 dos indivíduos identificados possuía entre 0 e 5 anos de idade. A prevalência de injúria nesse período etário foi de $12 \%$, sete crianças $^{(7)}$.

Diante do exposto, considerando os índices significativos das ocorrências de acidentes domésticos na primeira infância, o presente estudo emergiu da experiência do pesquisador na área de saúde da criança e de primeiros socorros. A vivência com esta prática suscitou o interesse em investigar os conhecimentos das mães de crianças da primeira infância sobre a prevenção de acidentes domésticos infantis

Esta pesquisa visa contribuir para a conscientização dos profissionais de saúde, sobretudo enfermeiros, acerca da ocorrência de acidentes domésticos com crianças da primeira infância, propiciando o desenvolvimento de programas e ações voltados para a diminuição da ocorrência de tais acidentes. Além disso, o presente estudo poderá servir como base de conhecimentos para o desenvolvimento de outras pesquisas e de tecnologias que objetivem a melhoria da proteção e da qualidade de vida dos menores em questão.

O objetivo geral do presente estudo foi identificar os conhecimentos das mães sobre a prevenção de acidentes domésticos infantis com crianças da primeira infância.

\section{METODOLOGIA}

Trata-se de um estudo descritivo-exploratório, com abordagem quantitativa. Foi realizado em um ambulatório de puericultura localizado no Campus do Pici da Universidade Federal do Ceará, na cidade de Fortaleza. Na época da coleta de dados havia 100 mães de crianças de zero a três anos cadastradas no serviço e a amostragem utilizada foi a por conveniência. A amostra foi constituída por 32 mães. Como critério de inclusão estabeleceu-se mães de crianças de zero a três anos frequentadoras do serviço de puericultura da referida unidade. Como critério de exclusão, mães que não tivessem condições psíquicas e emocionais para participarem da pesquisa.

A coleta de dados foi realizada no período de junho a agosto de 2015, às segundas, terças e quartas, no turno vespertino e na sala de espera do serviço.

Inicialmente, foi apresentado, às participantes, a finalidade do estudo e seu objetivo, explicando a importância do Termo de Consentimento Livre e Esclarecido - TCLE. Após a assinatura por parte do sujeito da pesquisa, era aplicado o instrumento de coleta.

Foi utilizado para coleta de dados um questionário semiestruturado contendo dois grupos de variáveis: variáveis sociodemográficas e econômicas das mães; e variáveis relacionadas ao perfil das crianças e a ocorrência de acidentes.

Para tabulação dos dados foi criado um banco de dados em planilha eletrônica para as análises descritivas, utilizando o programa Excel 2013. Os dados foram organizados em tabelas e gráficos e a análise ocorreu através do programa $\mathrm{Epi}^{\mathrm{Info}} \mathrm{IM}^{\mathrm{TM}}$ 7, por meio de freqüências absolutas e relativas.

Esta pesquisa foi aprovada pelo Comitê de Ética e Pesquisa da Universidade Federal do Ceará sob CAAE $n^{\circ}$ 32995214.9.0000.5054. Número do comprovante: 055180/2014 


\section{RESULTADOS}

Os dados são apresentados a seguir a partir dos dois grupos de variáveis. Desta forma, conforme as variáveis sociodemográficas e econômicas das mães (tabela 1), observou-se que metade das genitoras pertencem à faixa etária 22 a 29 anos. A maioria mora com o companheiro (58\%).

Das entrevistadas, $41,9 \%$ possuem ensino médio completo. Quanto à renda, 61,2\% recebem o valor mensal familiar de $\mathrm{R} \$$ 789 a 1576, o equivalente, em média, de um a dois salários mínimos. Em grande parte das residências moram quatro, cinco ou mais pessoas, representando $39,9 \%$.

No que se refere às variáveis relacionadas ao perfil das crianças e a ocorrência de acidentes, grande parte possui entre dois a seis meses (59,3\%). Constatou-se que 90,6\% das crianças não ficam sozinhas no domicilio, resultado que se mostrou bastante positivo. A maior parte das famílias relatadas possui apenas uma criança da primeira infância $(81,2 \%)$.

Um dado importante é o fato de que todas as mães abordadas tomam medidas preventivas contra a ocorrência de acidentes domésticos. De tais medidas, as que mais se destacam são o cuidado no armazenamento de produtos tóxicos e a vigilância da criança no espaço domiciliar, ambos com $24,9 \%$.

As genitoras foram questionadas quanto à ocorrência de acidentes domésticos com suas crianças. A maioria (62,5\%) inferiu que já ocorreram injúrias, evidenciando a susceptibilidade às lesões não intencionais, as quais requerem especial atenção. Acerca dos casos ocorridos, destacam-se as quedas $(66,6 \%)$.

Com relação à parte do corpo mais acometida pelos acidentes, $80 \%$ acometeram a cabeça/face. O local da casa em que mais ocorreram as injúrias foi o quarto, com $88,8 \%$ dos relatos. Acerca de quem estava acompanhando as crianças no momento do acidente, metade estavam na companhia dos pais.

\section{DISCUSSÃO}

\section{Das variáveis sociodemográficas e econômicas}

Com relação às variáveis sociodemográficas e econômicas das mães, o presente estudo evidenciou a metade das inquiridas como sendo da faixa etária de 20 a 29 anos. Em um estudo realizado com pais de 323 crianças, estas sendo menores de cinco anos de idade, no municipio de Teresina - PI, foi encontrado o mesmo perfil. Desta forma, podemos considerar que a maior parte das crianças menores de cinco anos está sob atenção de mães adultas jovens, o que pode significar menor conhecimento quanto à prevenção de acidentes, primeira experiência como cuidadoras e rotina ocupada com estudo e trabalho, deixando parte do cuidado e da atenção à criança a terceiros ${ }^{(8)}$

Além disso, abaixarendaestáassociadaproporcionalmente à ocorrência de acidentes domésticos, sugerindo uma influência da questão socioeconômica na incidência de tais eventos. Em uma pesquisa feita com cuidadores de crianças de $\mathrm{O}$ a 14 anos no município do Rio de Janeiro, a renda familiar também demonstrou ser um fator de influência proporcional ao índice de ocorrência de lesões não intencionais, uma vez que baixas condições socioeconômicas não proporcionam condições adequadas de lazer e de espaço para a criança brincar $^{(9)}$

\section{Quanto ao perfil das crianças e a ocorrência de acidentes}

Concernente às variáveis relacionadas ao perfil das crianças e a ocorrência de acidentes, a medida preventiva identificada como a mais utilizada para a prevenção de acidentes domésticos infantis foi o cuidado com o armazenamento de produtos tóxicos. Em um estudo realizado em um colégio privado no distrito de BragançaPortugal, que contou com a participação de 140 pessoas na condição de pais/ responsáveis de crianças de 0 a 10 anos de idade, essa é uma das medidas preventivas mais utilizadas por parte de tais inquiridos, com 19,6\% da totalidade das medidas praticadas. Isso mostra uma maior preocupação das mães com medicamentos e produtos tóxicos, havendo assim, a necessidade de mais atenção quanto ao método e as embalagens de armazenamento ${ }^{(10)}$.

A incidência de acidentes no presente estudo foi elevada, o que indica um número alto de tais eventos. Isso é confirmado em uma pesquisa realizada em Montes Claros - MG com 50 pais ou responsáveis de crianças de 0 a 12 anos, a maioria ${ }^{(26)}$ pertencendo à classe C (52,0\%) e 24 à classe D (42,0\%), aponta uma prevalência de 41 crianças acidentadas (82\%) do total de 50 estudadas ${ }^{(2)}$.

O presente estudo apontou que o tipo de acidente mais prevalente é a queda. Tal dado também é percebido em uma pesquisa em que 97 dos acidentes informados, 56,7\% deles foram quedas ${ }^{(2)}$. Em um estudo realizado a partir do atendimento em um hospital materno-infantil no sul do estado de Santa Catarina com 145 crianças de 0 a 14 anos vítimas de acidentes domésticos, a queda também representa o acidente mais evidente, com $55,2 \%$ dos casos $^{(11)}$.

As entrevistadas relataram que a grande maioria dos casos de acidentes ocorrem na cabeça/face, número que representa a maior ocorrência de lesões em tal parte do corpo. Tal fato não é encontrado no estudo supracitado realizado em Bragança-Portugal, que defende uma prevalência de 52,3\% dos casos nos membros, com cabeça/ face em segundo lugar, tendo $28 \%$ dos relatos ${ }^{(10)}$

Em relação a quem estava com as crianças no momento 
do acidente, o presente estudo indicou que em metade dos casos os menores estavam na companhia dos pais. Esse dado assemelha-se ao encontrado por Xavier-Gomes et al., em que pouco mais da metade dos acidentes ocorreram na presença dos pais. Percebe-se assim que a companhia dos pais apenas diminui o risco de acidentes, mas não impede que tais eventos ocorram $^{(2)}$.

Ainda que tenha chegado a resultados relevantes, pode ser apontada como limitação do estudo a pouca quantidade de pacientes na unidade em que foi feita a pesquisa.

\section{CONCLUSÃO}

O estudo demonstrou que há predominância de acidentes domésticos infantis em populações de baixa renda, de escolaridade média, de idade jovem e com várias pessoas morando na mesma residência. Percebeu-se a necessidade de serem fortalecidos conhecimentos junto as mães sobre prevenção de acidentes domésticos infantis, objetivando assim prevenir a ocorrência de injúrias, podendo ser criados para este fim programas e ações de prevenção.

Percebeu-se ainda que as abordagens prevencionistas não devem ser pautadas apenas nos agentes lesivos, levando em conta também fatores como, por exemplo, condições sociodemográficas, número de cômodos na casa, quantidade de habitantes no ambiente domiciliar e medidas preventivas tomadas, visto que as variáveis levantadas influenciam umas às outras, direta ou indiretamente, na ocorrência de acidentes domésticos.

\section{REFERÊNCIAS}

1. Hockenberry MJ, Wilson D. Fundamentos de Enfermagem Pediátrica. Rio de Janeiro: Edição 9, Editora Elsevier Brasil;2014.

2. Xavier-Gomes LM, Rocha RM, Andrade-Barbosa TL, Silva CSO Descrição dos acidentes domésticos ocorridos na infância. Mundo saúde (Impr.). 2013 [citado em 2017 mai 29]; 37(4): 394400. Disponivel em: http://www.saocamilo-sp.br/pdf/mundo_ saude/155558/A03.pdf.

3. Bezerra MAR, Rocha RC, Negreiros FS, Lira FMOM, Sousa LT, Santiago SCG. Acidentes domésticos em crianças: concepções práticas dos agentes comunitários de saúde. Cogitare enferm. 2014 [citado em 2017 mai 30]; 19(4): 776-784. Disponivel em: https://revistas.ufpr.br/cogitare/article/view/37301/23951.

4. Bivanco-Lima D, Moura JC, Tirico SHN, Mazzeo MR, Cunha MT, Sperandio RA et al. Promoção à saúde e prevenção de acidentes na infância: uma ação de estudantes de medicina. Rev. med. (São Paulo). 2013 [citado em 2017 jun 01]; 92(2): 119-127. Disponivel em: http://www.revistas.usp.br/revistadc/article/ view/79951/83885

5. Ramos ALC, Nunes LRM. Criança em ambiente doméstico/ familiar: consenso quanto aos fatores de risco de lesão não intencional. Referência. 2014 [citado em 2017 mai 0l]; 4(1):45-54. Disponivel em: http://www.scielo.mec.pt/pdf/ref/vserIVnl/serIVnla06.pdf.

6. Malta DC, Mascarenhas MDM, Neves ACM, Silva MA. Atendimentos por acidentes e violências na infância em serviços de emergências públicas. Cad. saúde pública. 2015 [citado em 2017 mai 01]; 31 (5):1095-1105. Disponivel em: http://www.scielo.br/ $\mathrm{pdf} / \mathrm{csp} / \mathrm{v} 3 \ln 5 / 0102-311 X-c s p-31-5-1095$.pdf.

7. André SB, Carvalho FM, Daltro CC, Pena P. Epidemiologia dos acidentes em uma comunidade de baixa renda em Salvador, Bahia. Rev. baiana saúde pública. 2014 [citado em 2017 mai 05];
38(3):585-597. Disponivel em: https://www.researchgate.net/ profile/Fernando_Carvalhol3/publication/295081784_Epidemiologia_dos_acidentes_em_uma_comunidade_de_baixa_ renda_de_Salvador_Bahia/links/57bbl96c08ae9fdf82efO229/ Epidemiologia-dos-acidentes-em-uma-comunidade-de-baixa-renda-de-Salvador-Bahia.pdf?origin=publication_detail

8. Brito MA, Melo AMN, Veras IC, Oliveira CMS, Bezerra MAR, Rocha SS. Fatores de risco no ambiente doméstico para quedas em crianças menores de cinco anos. Revista Gaúcha de Enfermagem. 2017 [citado em 2017 mai 18];38(3):1-9. Disponivel em: http://www.scielo.br/pdf/rgenf/v38n3/0102-6933-rgenf-38-3-e2017-001.pdf

9. Miranda IFA, Soares R, Torre K, Costa A, Fonseca T, Fernandes MG. Conhecimento dos responsáveis sobre a prevenção de acidentes domésticos envolvendo crianças. Revista de Pediatria SOPERJ. 2015 [citado em 2017 mai 28]; 15(1):6-12. Disponivel em: revistadepediatriasoperj.org.br/audiencia_pdf.asp?aid2=6388nomeArquivo.pdf.

10. Antão CCM, Mata MAP, Sousa MFG. Acidentes com crianças: perceção de pais, In: Jornadas de Enfermagem da Escola Superior de Saúde do IPB; 2013; Bragança-Portugal. Bragança: Primeiras Jornadas de Enfermagem da Escola Superior de Saúde do IPB; 2013 [citado em 2017 mai 28]. 40-48. Disponivel em: https://bibliotecadigital.ipb.pt/bitstream/10198/9376/3/ Ebook\%20Jornadas\%20Enfermagem\%202014\%2011\%203.pdf.

11. Copetti CL, Maciel GW, Daminelli CRT, Gualtieri PD, Souza RL Atendimentos a crianças e adolescentes vitimas de acidente domiciliar em um hospital materno infantil no sul de Santa Catarina. Revista Inova Saúde. 2014 [citado em 2017 mai 17]; 3(2):1-15. Disponivel em: http://periodicos.unesc.net/Inovasaude/article/ view/1310/1669. 